\title{
Remineralisation,
}

\section{whitening and prevention}

\section{By Ann Generlich,}

\section{senior PR and \\ marketing professional}

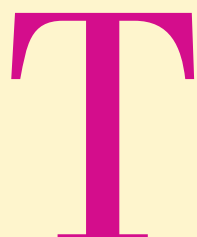

here are many problems

associated with the teeth and gums which become noticeably more pronounced as we age, including an increase in tooth wear,

sensitivity, staining and gum health problems.

Toothpastes and oral rinses containing hydroxyapatite, stabilised chlorine dioxide and fluoride could potentially help to combat the effects of ageing on the oral cavity and provide tooth health benefits.

Hydroxyapatite and fluoroapatite are both the hardest materials existing in nature and one of our major body components (comprising $60 \%$ of bone, $97 \%$ of tooth enamel and $70 \%$ of dentine).

Hydroxyapatite supports a naturally occurring function, the restorative role of saliva in the mouth, which is the first line of defence against caries. In oral hygiene products it can effectively improve the mechanism of regenerating tooth enamel and tangibly reduces the sensitivity of teeth by blocking dental tubules. ${ }^{1}$ Hydroxyapatite does this by neutralising acids caused by plaque bacteria ${ }^{2}$ and by providing calcium and phosphate ion building blocks, which diffuse back into the enamel to restore lost minerals. Hydroxyapatite also acts as a filler, repairing minute pits and fissures in the enamel surface, resulting in smoother, glossier enamel, with fewer sites likely to harbour plaque and stains. Hydroxyapatite has been shown to remineralise subsurface areas of the enamel and restores mineral density and translucency to enhance the whiteness of the teeth. ${ }^{3}$ It not only protects against decay by helping remove plaque, ${ }^{4}$ restores smoothness and mineral density to both surface and subsurface enamel, but it also makes teeth more resistant to acidic food and drinks and contributes to both their health and natural beauty.

Research suggests that dental caries might be triggered by three main factors: the host defence mechanism, the oral environment and bacteria. Plaque is often seen as the precursor to tooth decay, contributing to the oral cavity's overall dynamic environment that frequently undergoes rapid changes in $\mathrm{pH}$, nutrient availability and oxygen tension. 'Under suitable conditions, periodontal pathogens colonise the subgingival environment, incorporating into a tenacious biofilm, impacting both caries and periodontal disease' (Jorgsen and Slot, 2000). The plaque bacteria (Streptococcus mutans and Lactobacillus) demineralise the tooth surface by producing acid through the metabolisation of carbohydrates and therefore attack the minerals in the enamel, the cementum and the dentine. ${ }^{5}$ Acids produced by plaque bacteria lower the $\mathrm{pH}$ below 5.5 , seep into these channels and can dissolve carbonated hydroxyapatite and rods, causing the enamel to become demineralised. Tooth decay develops when the rate of demineralisation exceeds the rate of remineralisation, often linking to gum problems and tooth sensitivity.

The late periodontist, Professor Perry Ratcliff, maintained that the causes of periodontal disease are a combination of many processes, including activation of the immune system, alterations in connective gingival tissue metabolism, production of proteinases and cytokines and the destruction of host tissue by bacterial enzymes, along with many other factors. He claimed that extremely low concentrations of Volatile Sulphur Compounds (VSCs: by-products of the bacterial action and putrefaction in the oral cavity of debris such as stagnating food, rotting skin cells, toxins and other sulphur containing substrates), the family of gases which are primarily responsible for oral malodour, are also highly toxic to mouth tissues.

The challenge is to eliminate or control VSCs and bacteria in a way that does not introduce substances or cause bacteria to build an immune resistance. With its ability to help achieve optimal oral health, stabilised chlorine dioxide has proven to be extremely effective at this, as it works closely with the natural oral $\mathrm{pH}$. It acts as an oxidising agent to destroy amino acids to prevent their use as building blocks for protein (effectively assisting the control of biofilms and neutralising the production of acid in the mouth). Stabilised chlorine dioxide is very gentle on the delicate tissues of the mouth and decreases the ability of negative micro-organisms/bacteria in the oral cavity to multiply. Research has also shown that stabilised chlorine dioxide can demonstrate efficacy at lowering concentration of VSCs in periodontal pockets ${ }^{6}$ by changing the molecular structure on contact. It also does not have any unpleasant side effects. Stabilised chlorine dioxide not only provides an optimum aid for dental health and function, but based on its high patient compliance, helps encourage patients to become active participants in their own oral healthcare.

As the mouth provides a window into an individual's general health status, strategies to prevent dental problems have evolved over the years. With favourable behaviour towards remineralisation and prevention, oral hygiene products containing hydroxyapatite, stabilised chlorine dioxide and fluoride, such as the UltraDEX Recalcifying \& Whitening toothpaste and oral rinse, could be of value to a wide spectrum of clinical oral hygiene concerns, when used as part of an oral healthcare regime.

1. Lynch E, Brauer D S, Karpukhina N, Gillam D G, Hill R G. Multi-component bioactive glasses of varying fluoride content for treating dentin hypersensitivity. Dent Mater 2012; 28: 168-178.

2. Joiner A, Schäfer F, Hornby K et al. Enhanced enamel benefits from a novel fluoride toothpaste. Int Dent J 2009; 59: 244-253.

3. Niwa M, Sato T, Li W, Aoki H, Aoki $\mathrm{H}$, Daisaku T. Polishing and whitening properties of toothpaste containing hydroxyapatite. J Mater Sci Mater Med 2001; 12: 277-281.

4. Fujimara T, Arakawa T, Hayman R. Evaluation of cariogenic bacteria removal by nano-hydroxyapatite. 88th General Session \& Exhibition of the IADR. 14-17 July 2010.

5. Duckworth R M. The science behind caries prevention. Int Dent J 1993; 43: 529-539.

6. Ratcliff P, Johnson P. The relationships between oral malodor, gingivitis and periodontitis. J Periodontology 1999; 1-14. 